\title{
Evolutionary approach to optimisation of the operation of electric power distribution networks
}

\author{
Jan Stępień \\ University of Technology Kielce \\ Electrical Engineering Automatics and Computer Science \\ Faculty Kielce, Poland
}

\author{
Sylwester Filipiak \\ University of Technology Kielce \\ Electrical Engineering Automatics and Computer Science \\ Faculty Kielce, Poland
}

\begin{abstract}
An idea of using a classifying system and coevolutionary algorithm for operation support of electric power distribution systems operators has been presented in the paper. The method proposed by the author of the work is typified by the short time of designating the most rational post breakdown configurations in complex electric power Medium Voltage distribution network structures. It is the use by the classifying system working with the co-evolution algorithm that enables the effective creation of substitute scenarios for the Medium Voltage electric power distribution network. The method drawn up may be used in current systems managing the work of distribution networks to assist network operators in taking decisions concerning connection actions in supervised electric power systems.
\end{abstract}

Keywords-evolutionary algorithms; distribution power networks; electric breakdown

\section{INTRODUCTION}

Distribution networks faults can cause power failure for many users, what can be a reason of major economical losses. In the literature on the distribution systems operation the problem of power delivery recovery in case of the network failure is one of the very important aspects of a proper operation of the distribution systems. Planning a restoration service for distribution systems is a critical task for dispatchers in a control center. Restoration attempts to supply an ample amount of power to nonfaulty out-of-service areas for as many customers as possible while safely operating the distribution system. Reconfiguration is the process of changing the open/closed status of switches and is done for volt/var support, loss reduction, load balancing and restoration. Reconfiguration for restoration is a combinatorial problem involving searching an enormous space of solutions. The problems with integer variables are NP hard, meaning no known algorithm exists to solve these problems in polynomial time. However, reconfiguration for restoration problem is both NP hard and NP and hence belongs to the class of NP complete problems. For such kind of problems, the solution time increases with an increase in the number of integer variables. However, the solution time generally depends on the formulation.

The aim of the research is to develop a method that uses the classifying system and co-evolutionary algorithm to determine, for the assumed conditions, the most profitable distribution network configuration. The important feature of the method is the possibility to form the substitute network configuration with the use of information coming from the simulated network operation states, where the information on reliability parameters of the network or exploitation periods of the network elements can be also exploited.

\section{METHODS USED}

First, Many approaches have been proposed to solve the restoration problem from different perspectives. For instance, researchers [1, 2] incorporated dispatcher's experience and operating rules into an expert system to assist the dispatcher. Related investigations formulated the restoration problem as an optimization problem to minimize the number of unserviced customers $[3,4]$. This problem has been approached using heuristics [5, 6, 7] mathematical programming [8], metaheuristics (genetic algorithms, tabu search, simulated annealing) $[9,10]$ and expert systems $[11]$.

In works $[12,13,14]$ are presented methods concerning the use revolution algorithms drawn up to resolve multi-criteria problems in optimising electric power networks. These methods concern the development of specialised means of coding, reproduction methods based on domination and also use of co-evolutionary approaches. Several evolutionary algorithms have been developed to deal with distribution system reconfiguration problems $[15,16,17,18]$. Although the obtained results have been encouraging, the majority of evolutionary algorithms still demand high running time when applied to large-scale distribution systems. In [14], it was shown that the tree encoding (data structure) used is a critical factor for the performance of evolutionary algorithms applied to such large distribution systems. Other critical aspects of distribution systems are the genetic operators that are implemented. Generally these operators do not generate radial configurations [19]. In order to improve the performance obtained by evolutionary algorithms in distribution system reconfiguration problems, a tree encoding based on graph chains, called graph chains representation, and its corresponding genetic operators were developed in [14]. These operators produce only radial configurations. Although the requirement of a radial configuration is common for distribution system reconfiguration problems, it makes the network modeling more difficult to efficiently reconfigure distribution systems.

In the article the author presented the results of his works concerning the method drawn up using the system classifying cooperation with the co-evolutionary algorithm, in order to assist the work of electrical energy distribution systems operators. The elaborated method uses the classifying system to 
determine, for the assumed conditions, the most profitable distribution network configuration. The important feature of the method is the possibility to form the substitute network configuration with the use of information coming from the simulated network operation states, where the information on reliability parameters of the network or exploitation periods of the network elements can be also exploited.

Cooperation of the co-evolutionary algorithm with the classification system (drawn up by the author of the work) enables significant reduction of the classification time (reduces the iterative calculation process on average by $40 \%$ ), which is significant from the practical point of view in the application of this method in current systems of distribution network operation management. The application of a classification system to the analysed task also enables improvement of the effectiveness of the performance process of designating the scenario of the substitute network configurations. Improvement of the efficiency of the network configuration designation process is obtained using the sought information (with use of the announcement creation process), in the collections of classifiers to create sub-populations of solutions for the coevolutionary algorithm, which would be used to search for the collection of Pareto-optimal solutions. The process of creating a collection of classifiers describing the substitute network configuration was performed by the author supported by the theoretical genetic basics of self-teaching system. Classifiers may be created (for analysed network structure) for the most probable break down situations, which arise from regarding the stage of choice of the simulated break down situations (in the analysed network) reliability characteristics and the usage durations of network elements. The result of the works performed is the drawing up of an effective method enabling the rapid designation of substitute network configurations, also for very complex network structures. The method may be used in information systems for the current operation management of electric power distribution networks..

\section{A METHOD USING THE ClASSIFIER SYSTEM}

\section{A. Evaluating system in the elaborated method}

The classifier system is a system that learns the syntactic simple rules in order to co-ordinate its actions in any environment and includes the three basic components [20, 21]: rule and message system, evaluating system, evolutionary algorithm. In the classifier system the information from the outer environment is processed into the messages of a given format. The messages are further placed on the message list, where they can activate the classifiers.

In the elaborated method (based on the classifier system idea) known procedures, performing message processing or classifier evaluation have been used. Certain modifications resulting from the specificity of the considered problem have been introduced:

- the message about the fault is described in the form: a list with numbers of not supplied nodes, and a list with numbers of fault elements :

$<$ message $>::=($ numbers of not supplied nodes $)+($ numbers of fault elements)
- in the classifier notation following syntax has been taken into account in the notation actually used:

$\langle$ classifier $>::=\langle$ condition $>:\langle$ message $>$

$<$ classifier $>::=<$ numbers of not supplied nodes + numbers

of fault elements $>:\langle$ post-fault configuration>

With regard to the specific character of the analysed task (concerning breakdown of elements in the network structure) the author has drawn up a modified announcement processing procedure (describing network break down situations). In the suggested method the announcement processing process and the evaluation of classifiers is divided into two stages described below.

Step 1 consists of the search in the collections of classifiers for such, for which the conditions are compatible with the announcement describing the existing network breakdown. Comparison of the announcement (containing information about damaged network elements and of network nodes deprived of current supply) with the conditions of classifiers enables the search and activation of classifiers containing coded information about network configurations, in which there are no damaged elements. Conformity of the announcement with the conditions of the classifiers in the first stage of the suggested method is defined on the basis of comparison of the numbers of network nodes without power supply recorded in the announcement, with the numbers of network nodes recorded in the first part of the classifiers (which corresponded to the concealed zeros on the appropriate positions of communication code tracts and classifier). After searching for classifiers conforming to the announcement the evaluation takes place of the so-called offer of these classifiers. The classifier distinguished by the highest offer was next used as a following announcement.

Step 2 concerns the search for classifiers whose conditions will be according to the announcement of the classifiers designated in stage 1 . The conformity of the announcement with the conditions of the classifiers in this stage was defined on the basis of the differences between the power supply routes of the chosen line sections (from the list of network nodes deprived of power supply) with the configuration recorded in the announcement and classifiers.

With regard for these specific natures of the analysed task the author suggested a two-part description of the announcement (describing the breakdown situation of the network). The first part of the announcement is recorded as a length of zero-ones relating to the number of elements equal to the number of network line sections of the analysed network. Value 1 on the defined position corresponding to the number of the network length, indicates length with power supply, and 0 indicates network node without power supply as a result of breakdown. The second part of the announcement contains information about the damaged elements and also information about the configuration of network elements. For the description of this part of the announcement the author introduced the following marking notation: 0 - means damaged element, 1 - means actually used element, \#- means element remaining in reserve. Below is showing an example of the process of creating announcements for the breakdown status of 
the electric power network system (composed of a small number of elements), the structure of which is reflected in graphic form on drawing 1. For the network graph from drawing 1, the case is examined of a breakdown on line $l 4$.

The performance of the process of creation of announcements enables search in the collection of classifiers for information, the use of which assists the process implemented by the co-evolutionary algorithm.

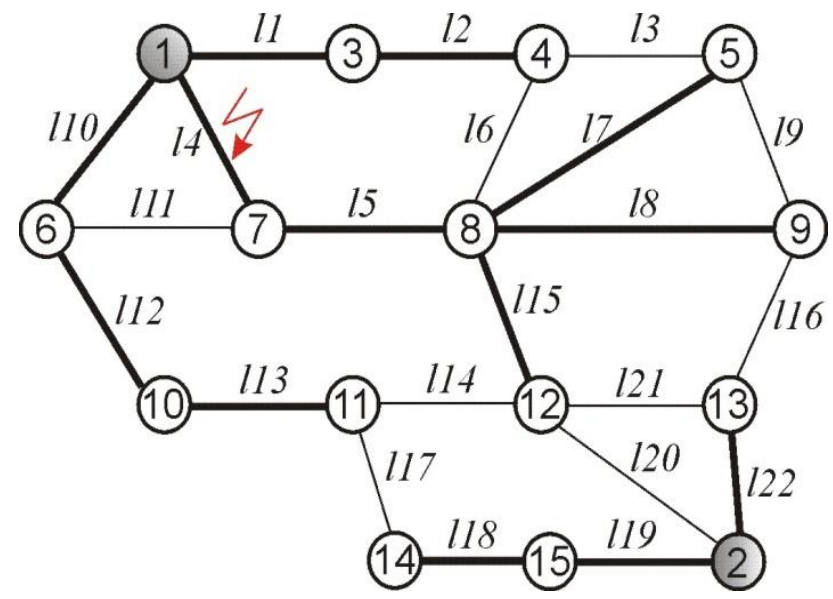

Fig. 1 Graph of the analysed distribution network

The announcement describing the considered breakdown status was described as follows:

\section{message 1}

111101000110111 | 11\#0\#\#\#\#\#1\#11\#\#\#\#11\#\#1

The sought for classifiers in the first stage (fig.2): classifier 1

111101100110111 | $11110 \# \# \# 11 \# 1111 \# \# 11$ \#\#1 classifier 2

111101111111111 | $11111 \# 01 \# 1 \# 11 \# 1 \# \# 11 \# \# 1$

In the initial part of calculations a message on the fault occurring in the network is being read. Procedures verifying the matching between the classifiers and the generated message are performed subsequently and then the classifiers are assessed.

The strength $S$ of the classifier, which has shown the best bid in the so-called auction process, is increased by the reward given by the system. Simultaneously its strength is decreased by the value of the bid given by the classifier. The rule and message procedures perform the process of classifiers checking and evaluation, in aspect of using the information contained in them for solving the problematic situations. This allows for appointing of the group of classifiers containing the useful information on the searched post-fault network configuration.

The bid of the best classifier increases the strength of other active classifiers proportionally to their bids. Moreover, the strength of all the active classifiers is decreased by a certain, determined value. The effective bid value has been calculated in a following way [20,21]:

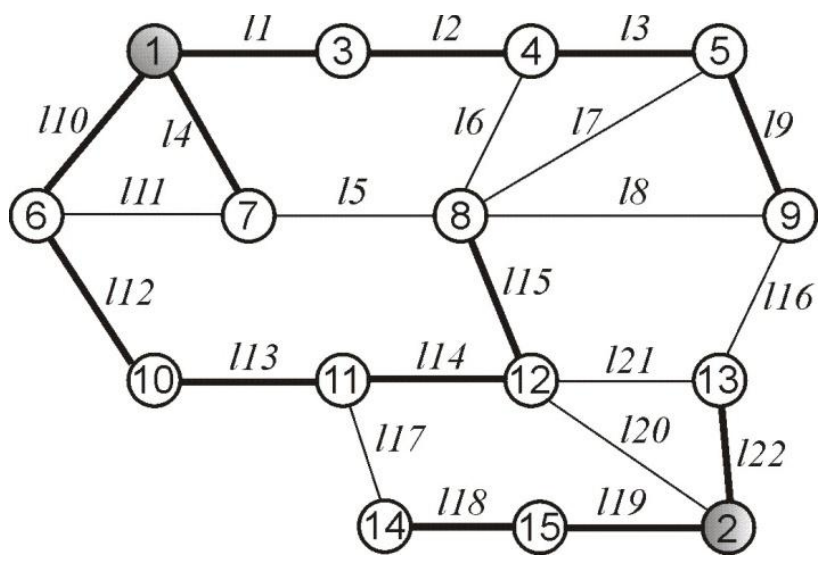

Fig. 2 Graph of the analysed distribution network for classifier 1

$$
\begin{gathered}
S_{i}(t+1)=S_{i}(t)-c_{b i d} \cdot S_{i}(t)-c_{t a x} \cdot S_{i}(t)+r(t) \\
B_{i}=c_{b i d} \cdot\left(e_{b i d 1}+e_{b i d 2} \cdot S p_{i}\right) \cdot S_{i} \\
E B_{i}=c_{b i d} \cdot\left(e_{b i d 1}+e_{b i d 2} \cdot S p_{i}\right) \cdot S_{i}+e_{b r}
\end{gathered}
$$

where: $B_{i}$ - bid value of the $i$-th classifier, $E B_{i}$ - effective bid value of the $i$-th classifier, $S p_{i}$ - specificity of the $i$-th classifier, $S_{i}$ - strength of the $i$-th classifier, $c_{b i d}$ - investment coefficient $\left(c_{b i d}=0,1\right), e_{b i d l}, e_{b i d 2}-$ coefficients of the classifier linear specificity function $\left(e_{b i d l}=0,65, e_{b i d 2}=0,35\right), e_{b r}-$ random value generated with the use of a normal distribution generator, $c_{t a x}$ turnover tax coefficient $c_{\text {tax }}=001, r$ - coefficient of reward paid for the best classifier $\mathrm{r}=2$.

\section{B. Co-evolutionary algorithm}

To modifications of the evolutionary algorithm enabling solutions of multi-criteria tasks are counted among others the application of the co-evolutionary approach. Application of the co-evolutionary algorithm to the analysed task creates $m$ population; in each of them the adaptation function is defined on the basis of another component quality indicator vector. After successive performance (population supplementation with new elements), and through renewed reproduction, these populations are connected, and then were again divided so that each population elements may attain an unlimited population. The sought-after solution is the Pareto-optimal collection of solutions.

To encode the individuals representing various network configuration variants in a form of a sparse graph, the bequest of chromosomes in the form of a vector of inversion has been assumed. Each component of the vector of inversion, corresponding to the number of the graph node, is equal to the number of the supplying node. A well-known roulette selection method on the remaining fractional part has been used as a selection method. Two specialised reconfiguration operators have been used in the algorithm to create new solutions (crossover probability $\mathrm{pk}=0,95$, mutation probability $\mathrm{pm}=0,15$ ). In the presented calculation method creating of new variants of the analysed problem solutions has been realised according to the following procedure:

1) Selection of two network configuration variants from the current population (recorded in the vectors of inversion), 
2) Node selection from the list of nodes with no supply,

3) Rewriting of the supply routes of the formerly selected node from the vector selected in step 1 to the auxiliary table,

4) Roulette selection of the node from the created table,

5) Rewriting of the further part of the supply route from the second vector, starting from the node selected in step 4, to the second of the selected vectors.

The aim of using of this kind of operator, creating new variants of distribution network configuration, was to examine the change variants effectiveness in the part of the networks close to the supply points, as well as in parts of the analysed network system affected by failures.

In order to obtain proper solutions following limiting constraints resulting from technical requirements for proper operation of the distribution network have been taken into account:

- Not exceeding of the maximum transmission currents of the line sections,

- Not exceeding of the allowable voltage drops in the network nodes supply routes,

On the base of the source data $[18,20]$ and own research following values of significant parameters of the calculation system have been assumed in the calculation procedures: number of classifiers $n=200$, crowding factor for classifier population $\mathrm{cs}=3$.

\section{Assumed optimization criteria}

Following criteria have been assumed substantial for the optimisation problem of post-fault network configuration:

- Minimisation of the number of switching activities leading to obtaining a substitute network configuration:

$$
\operatorname{Min}_{j} u_{1}\left(X_{j}\right)=n_{j}-n_{0} \quad \text { Where } j=1,2, \ldots, m
$$

where: $X_{j}$ - vector containing information on the $j$-th variant of the distribution network configuration, $m$ - number of solution variants, $n_{j}$ - number of switching activities, $n_{0}$ number of switching activities in the basic configuration.

- Maximal level of reliability of supply of electric power to recipients:

$$
\operatorname{Min}_{j} u_{2}\left(X_{j}\right)=\min \left\{\max \left(1-p_{i k}\right)\right\}
$$

Where: $p_{i k}$ - coefficient of reliability of supply track $i$-th $\mathrm{w}$ of recipient node designated for $\mathrm{k}$-th of year of cable exploitation,

- Minimisation of the voltage deviation in the network nodes:

$$
\operatorname{Min}_{\mathrm{j}} \mathrm{u}_{3}\left(X_{j}\right)=\max _{i}\left(\frac{U_{i}}{U_{N}} \cdot 100\right)
$$

Where: $U_{N}$ - distribution network nominal voltage, $U_{i}-$ voltage value in the $i$-th user node of the network,
- Minimisation of the power load degree coefficient of the found group of the most loaded network elements.

$$
\operatorname{Min}_{j} u_{4}\left(X_{j}\right)=\max _{k} \frac{\sum_{i=1}^{n} P_{\max , i}}{n}
$$

Where: $k$ - the number of power supply route network nodes of the reception network, $n$ - the number of the most heavily loaded network elements.

- Minimisation of the technical losses in the distribution systems:

$$
\operatorname{Min}_{j} u_{5}\left(X_{j}\right)=\min \left\{\sum_{i=1}^{g}\left(\Delta P_{i}+k_{e} \cdot \Delta Q_{i}\right)\right\}
$$

Where: $\mathrm{g}$ - number of sections underload in given network configuration variant, $\Delta P_{i}$ - value of loss of power in i-th network section, ke - electric power equivalent of passive power.

The assumed membership functions used for the main variables description have been defined as follows:

$$
u_{f_{i}}(X)=\left\{\begin{array}{lc}
1, & \text { if } \quad f_{i}(X) \leq f_{i}^{\min } \\
\left(\frac{f_{i}^{\max }-f_{i}(X)}{\left.f_{i}^{\max }-f_{i}^{\min }\right),}\right. & \text { if } \quad f_{i}^{\min }<f_{i}(X) \leq f_{i}^{\max } \\
0, & \text { if } \quad f_{i}^{\max }<f_{i}(X)
\end{array}\right.
$$

\section{CASe Studies}

The pre-analysed calculation problem concerns the designation of the supply network configuration for the breakdown operations statuses of the network, arising from damaged network elements, their loading and also the exceeding of permissible voltage deviations in network line sections. Considered breakdown status busbars main supply station number 6 . The consequence of this breakdown is lack of power supply for a significant part of the network nodes. The sought-after solution is a substitute network configuration enabling the restoration of power supplies to as great a number of recipient network nodes as possible. The considered task is a multi-criteria optimisation task. In the presented graph (fig. 3) the filled nodes symbolise the main supplying points, whereas the bold branches symbolise the elements taking part in the load transfer.

For the below considered breakdown situation in the analysed network, which is composed of 556 network nodes the author accepted the abbreviated description of announcements and also classifiers. The abbreviated description however contains instead of the zero-one tract (part of the first announcement) the numbers of line sections deprived of power, whereas as part of the second announcement the numbers of damaged elements are given. In the elaborated calculation model a so-called vector of inversion has been used for the network configuration description. As a result of the accomplishment of the first stage of the process the announcement creation for the searched for classifier is 
shown in table 1 . In the column relating to network configurations noted in the inversion vector only the initial and

final elements of this vector are noted.

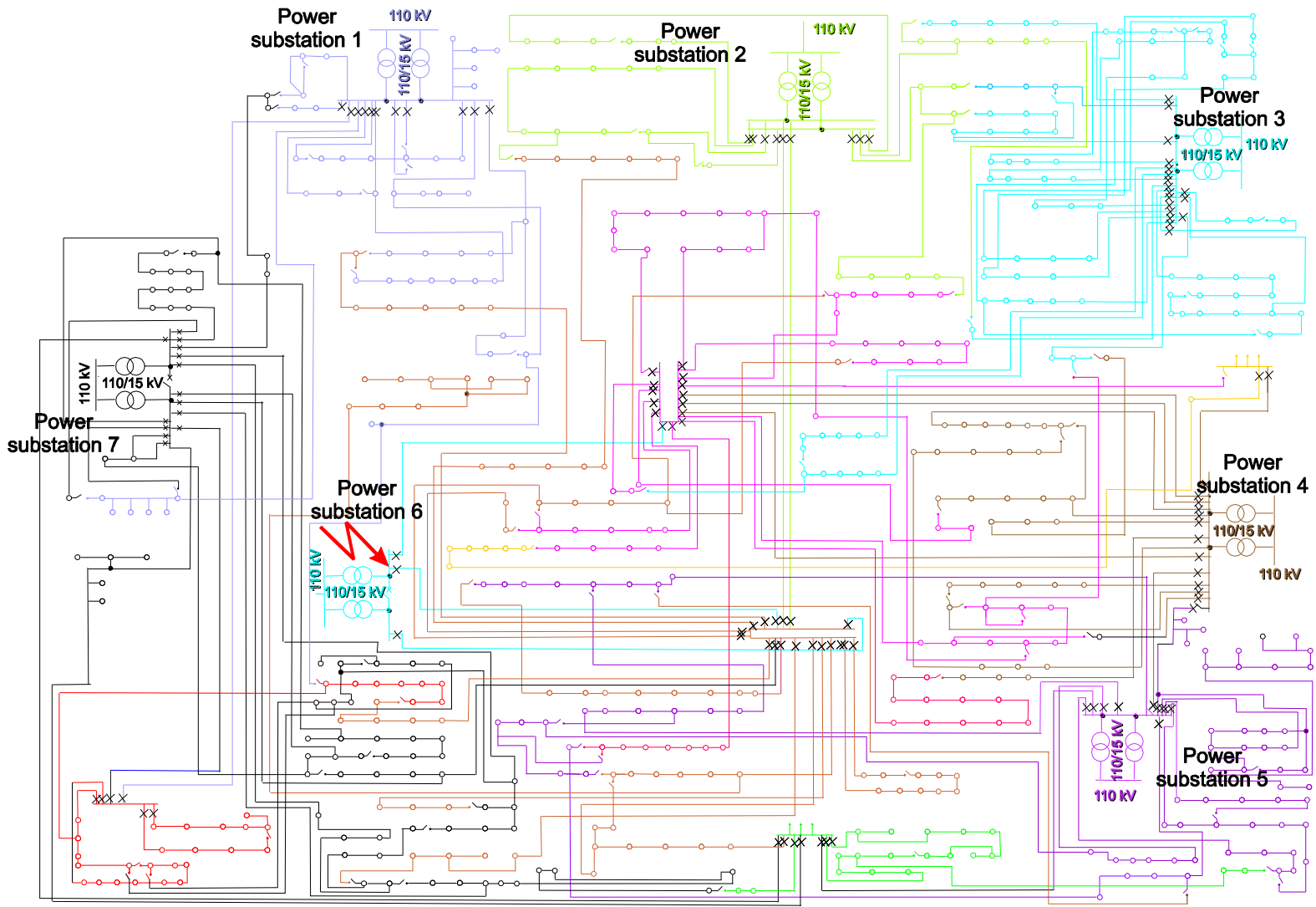

Fig. 3 Diagram of the analysed system of the medium voltage distribution network

TABLE I. ACTIVE CLASSIFIERS

\begin{tabular}{|c|c|c|c|c|c|}
\hline No & $\begin{array}{c}\text { condition: } \\
\text { (numbers of non- } \\
\text { supplied nodes) and } \\
\text { (numbers of fault } \\
\text { elements) }\end{array}$ & $\begin{array}{c}\text { answer of } \\
\text { system } \\
\text { (recorded in } \\
\text { the vectors of } \\
\text { inversion) } \\
\end{array}$ & $\begin{array}{c}S_{i}- \\
\text { strength } \\
\text { of the } i- \\
\quad t h \\
\text { classifier }\end{array}$ & Bid & $\begin{array}{c}S_{i}- \\
\text { strength } \\
\text { of the } i- \\
\quad t h \\
\text { classifier }\end{array}$ \\
\hline 1. & $\begin{array}{l}(8,22,28,29,31,73,1 \\
78,195,291 \ldots, 188) \\
\text { and }\left(6 \_8\right)\end{array}$ & $\begin{array}{l}\mathrm{x}, \mathrm{X}, \mathrm{x}, \mathrm{x}, \mathrm{X}, \mathrm{x}, \mathrm{x}, \\
4,6,5,1,13,14, \\
\ldots \\
554,7\end{array}$ & $S_{1}=10$ & $\begin{array}{c}\mathrm{B}_{1}= \\
0,813 \\
\mathrm{~EB}_{1}= \\
0,802\end{array}$ & $\begin{array}{c}S_{1}=10+ \\
0,622\end{array}$ \\
\hline 2. & $\begin{array}{l}(9,16,20,21,57,80,8 \\
1,101,117, \ldots, 363) \\
\text { and }\left(6 \_9\right)\end{array}$ & $\begin{array}{l}\mathbf{x}, \mathbf{x}, \mathbf{x}, \mathbf{x}, \mathbf{x}, \mathbf{x}, \mathbf{x}, \\
\mathbf{6 , 2 , 5 , 7 , 1 3 , 1 4}, \\
\ldots \\
, \mathbf{5 5 4 , 7}\end{array}$ & $S_{2}=10$ & $\begin{array}{c}\mathbf{B}_{2}= \\
0,823 \\
\mathbf{E B}_{2}= \\
0,831 \\
\end{array}$ & $\begin{array}{c}S_{2}=10+ \\
2\end{array}$ \\
\hline 3. & $\begin{array}{l}(29,73,72,71,70, \\
69,68) \\
\text { and }\left(8 \_29\right)\end{array}$ & $\begin{array}{l}\mathrm{x}, \mathrm{x}, \mathrm{X}, \mathrm{x}, \mathrm{x}, \mathrm{x}, \mathrm{x}, \\
6,6,5,1,13,14, \\
\ldots \\
554,7\end{array}$ & $S_{2}=10$ & $\begin{array}{l}\mathrm{B}_{2}= \\
0,639 \\
\mathrm{~EB}_{2}= \\
0,637\end{array}$ & $\begin{array}{c}S_{3}=10+ \\
0,211\end{array}$ \\
\hline
\end{tabular}

According to the idea of classifying systems through the process of announcement creation, then follows the evaluation of the revealed classifiers, which consists of the calculation of the so-called offer of the classifiers being the measure of their suitability to resolve the analysed task. As a result of the performance of the process of the creation and evaluation of announcements executed in the first stage, as the classifier with the best offer is defined as classifier number 2. This classifier served to create the announcement of the following stage of classifiers search.

TABLE II. ACTIVE CLASSIFIERS AFTER PROCESS EVALUATING

\begin{tabular}{|c|c|c|c|c|c|}
\hline No & $\begin{array}{l}\text { condition: } \\
\text { (numbers of } \\
\text { non-supplied } \\
\text { nodes) and } \\
\text { (numbers of } \\
\text { fault elements) }\end{array}$ & $\begin{array}{l}\text { answer of system } \\
\text { (recorded in the } \\
\text { vectors of } \\
\text { inversion) }\end{array}$ & $\begin{array}{c}S_{i}- \\
\text { strength } \\
\text { of the } i \text { - } \\
\text { th } \\
\text { classifier }\end{array}$ & Bid & $\begin{array}{c}S_{i-} \\
\text { strength } \\
\text { of the } i \text { - } \\
\text { th } \\
\text { classifier }\end{array}$ \\
\hline 1. & $\begin{array}{l}(99,16,20,21,5 \\
7,80,81,101,11 \\
7, \ldots, 363) \text { and } \\
\left(2 \_9\right)\end{array}$ & $\begin{array}{l}\mathbf{x}, \mathbf{x}, \mathbf{x}, \mathbf{x}, \mathbf{x}, \mathbf{x}, \mathbf{x}, \mathbf{6}, \mathbf{2} \\
\mathbf{5 , 7 , 1 3 , 1 4 , 1 5 , 1 6 , 9} \\
\cdots \\
, \mathbf{5 5 4 , 7}\end{array}$ & $S_{1}=10$ & $\begin{array}{c}B_{1}= \\
0,941 \\
E B_{1}= \\
0,975\end{array}$ & $S_{1}=10+2$ \\
\hline 2. & $\begin{array}{l}(10,413,414,41 \\
9,432,412,415, \\
\ldots 438) \text { and } \\
\left(7 \_10\right)\end{array}$ & $\begin{array}{l}\mathrm{x}, \mathrm{x}, \mathrm{x}, \mathrm{x}, \mathrm{x}, \mathrm{x}, \mathrm{x}, 6,2, \\
5,7,13,14,15,16,9 \\
\ldots \\
, 554,7\end{array}$ & $S_{2}=10$ & $\begin{array}{c}B_{2}= \\
0,712 \\
E B_{2}= \\
0,732\end{array}$ & $\begin{array}{r}S_{2}=10+ \\
+0,975\end{array}$ \\
\hline
\end{tabular}

As a result of the performance of the announcement creation process and the evaluation of active classifiers, information was obtained, which might be used to create a population (size 100) of solution variants subsequently created by the co-evolutionary algorithm. This algorithm is based simultaneously on 5 subpopulations, from which each evaluation was the basis for another adaptation function 
(dependencies 4 to 8 ). The sought-after solution in this case is a collection of solutions in the form of alternative configurations of the analysed network. The course of the process designating the best solutions in subpopulation 3 and 4 is shown in drawings 4 and 5 . Figures 4 b and 5 b show the realisation of the calculation process, which used information for active classifiers.

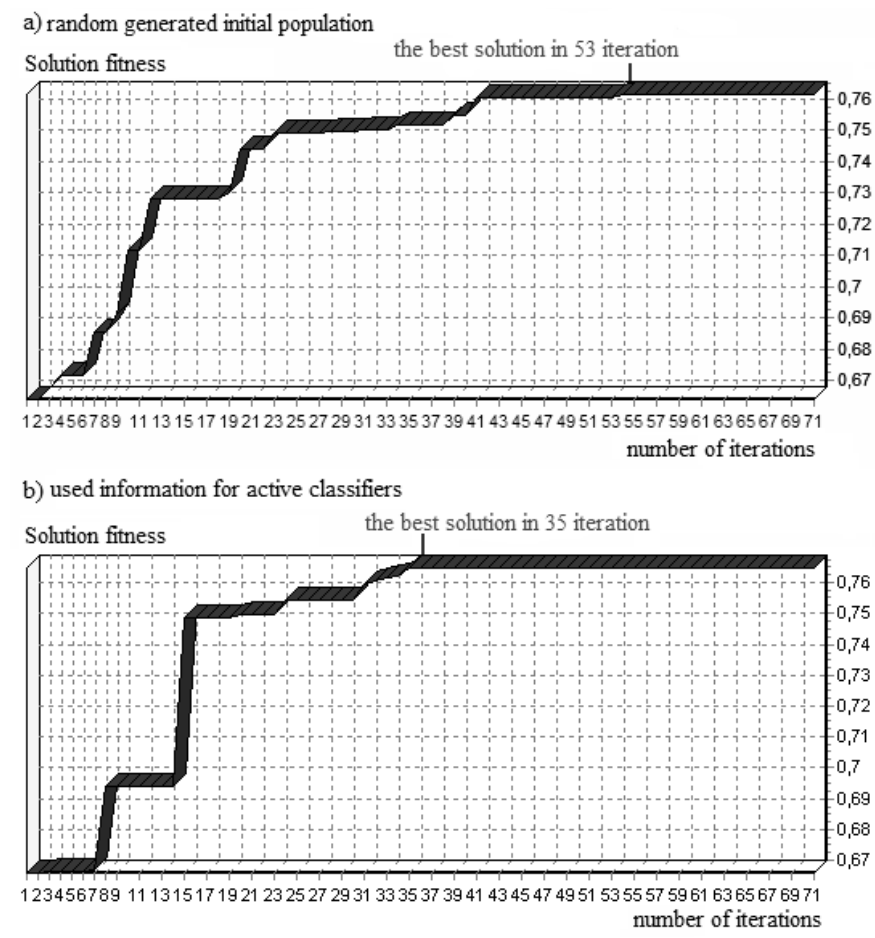

Fig. 4 Example of best solutions fitness waveform in subpopulation number 4

Cooperation of the co-evolutionary algorithm with the classification system enables significant reduction of time of obtainment of solutions (reduces the iterative calculation process on average by $40 \%$ ), which is significant from the practical point of view in the application of this method in current systems of distribution network operation management.

Information on the best solutions in subpopulations no. 3 is shown in graphic form on drawing 6. As a solution to the task of designating a substitute network configuration in the event of a breakdown of the analysed distribution network, obtained with the use of co-evolution algorithm the best solution variants is accepted from 5 subpopulations. Information concerning the best obtained solutions is shown in table 3.

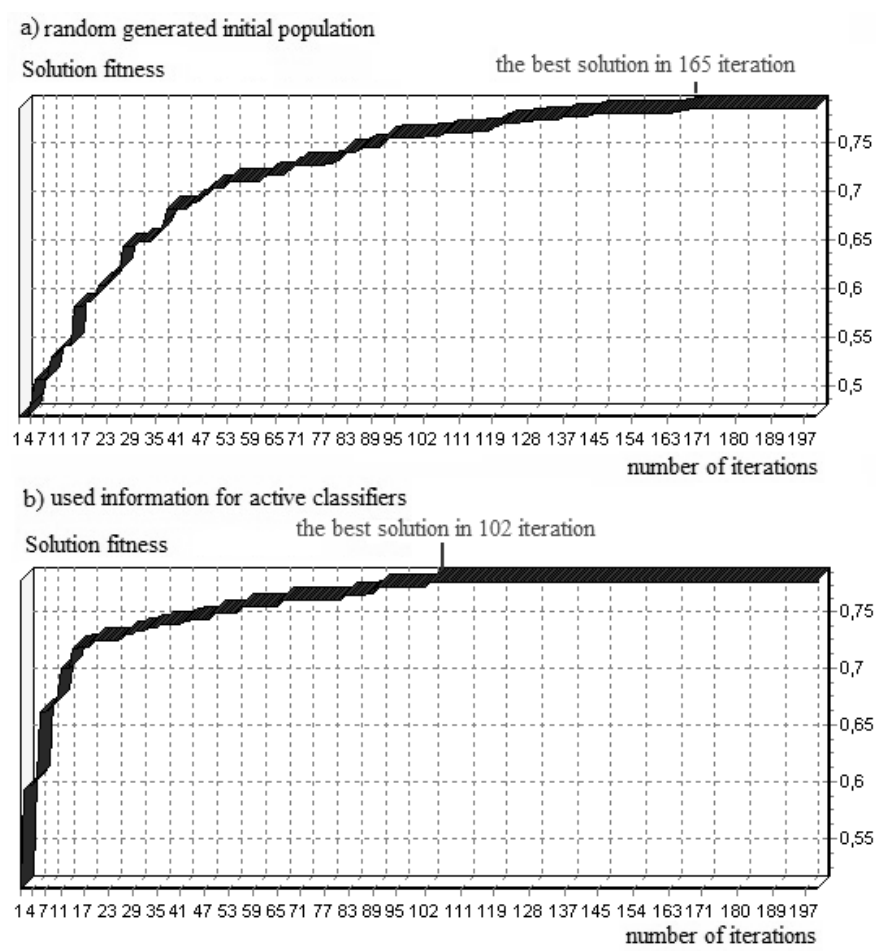

Fig. 5 Example of best solutions fitness waveform in subpopulation number 4

TABLE III. RESULTS ARE SHOWN OF CALCULATIONS FOR SOUGHT-AFTER POST BREAKDOWN CONFIGURATION OF ANALYSED NETWORK

\begin{tabular}{|c|c|c|c|c|c|}
\hline No & $\begin{array}{c}\text { Results for } \\
\text { subpopulation } \\
1\end{array}$ & $\begin{array}{c}\text { Results for } \\
\text { subpopulation } \\
2 \\
\end{array}$ & $\begin{array}{c}\text { Results for } \\
\text { subpopulation } \\
3\end{array}$ & $\begin{array}{c}\text { Results for } \\
\text { subpopulation } \\
4\end{array}$ & $\begin{array}{c}\text { Results for } \\
\text { subpopulation } \\
5\end{array}$ \\
\hline 1 & $\begin{array}{c}\mathrm{L}_{\mathrm{pz}}=4 \\
\mathrm{u}_{1}(\mathrm{X})=0,893\end{array}$ & $\begin{array}{c}\mathrm{L}_{\mathrm{pz}}=8 \\
\mathrm{u}_{1}(\mathrm{X})=0,671\end{array}$ & $\begin{array}{c}\mathrm{L}_{\mathrm{pz}}=7 \\
\mathrm{u}_{1}(\mathrm{X})=0,707\end{array}$ & $\begin{array}{c}\mathrm{L}_{\mathrm{pz}}=10 \\
\mathrm{u}_{1}(\mathrm{x})=0,641\end{array}$ & $\begin{array}{c}\mathrm{L}_{\mathrm{pz}}=12 \\
\mathrm{u}_{1}(\mathrm{x})=0,619\end{array}$ \\
\hline 2 & $\begin{array}{l}\mathrm{p}=0,998031 \\
\mathrm{u}_{2}(\mathrm{x})=0,896\end{array}$ & $\begin{array}{l}\mathrm{p}=0,998381 \\
\mathrm{u}_{2}(\mathrm{x})=0,998\end{array}$ & $\begin{array}{l}\mathrm{p}=0,998152 \\
\mathrm{u}_{2}(\mathrm{x})=0,943\end{array}$ & $\begin{array}{l}\mathrm{p}=0,997752 \\
\mathrm{u}_{2}(\mathrm{x})=0,790\end{array}$ & $\begin{array}{l}\mathrm{p}=0,998261 \\
\mathrm{u}_{2}(\mathrm{x})=0,985\end{array}$ \\
\hline 3 & $\begin{array}{l}\delta \mathrm{U}=2,23 \% \\
\mathrm{u}_{3}(\mathrm{x})=0,647\end{array}$ & $\begin{array}{c}\delta U=1,22 \% \\
u_{3}(x)=0,752\end{array}$ & $\begin{array}{c}\delta \mathrm{U}=1,18 \% \\
\mathrm{u}_{3}(\mathrm{x})=0,765\end{array}$ & $\begin{array}{c}\delta \mathrm{U}=1,31 \% \\
\mathrm{u}_{3}(\mathrm{x})=0,734\end{array}$ & $\begin{array}{c}\delta \mathrm{U}=1,22 \% \\
\mathrm{u}_{3}(\mathrm{x})=0,752\end{array}$ \\
\hline 4 & $\begin{array}{l}\Delta \mathrm{P}=2895 \mathrm{~kW} \\
\mathrm{u}_{4}(\mathrm{x})=0,635\end{array}$ & $\begin{array}{l}\Delta \mathrm{P}=2679 \mathrm{~kW} \\
\mathrm{u}_{4}(\mathrm{x})=0,667\end{array}$ & $\begin{array}{l}\Delta \mathrm{P}=2675 \mathrm{~kW} \\
\mathrm{u}_{4}(\mathrm{x})=0,670\end{array}$ & $\begin{array}{l}\Delta \mathrm{P}=2561 \mathrm{~kW} \\
\mathrm{u}_{4}(\mathrm{x})=0,740\end{array}$ & $\begin{array}{c}\Delta \mathrm{P}=2654 \mathrm{~kW} \\
\mathrm{u}_{4}(\mathrm{x})=0,682\end{array}$ \\
\hline 5 & $\begin{array}{l}\mathrm{k}_{\mathrm{obc}}=0,669 \\
\mathrm{u}_{5}(\mathrm{x})=0,743\end{array}$ & $\begin{array}{l}\mathrm{k}_{\mathrm{obc}}=0,584 \\
\mathrm{u}_{5}(\mathrm{x})=0,886\end{array}$ & $\begin{array}{l}\mathrm{k}_{\mathrm{obc}}=0,577 \\
\mathrm{u}_{5}(\mathrm{x})=0,898\end{array}$ & $\begin{array}{l}\mathrm{k}_{\mathrm{obc}}=0,771 \\
\mathrm{u}_{5}(\mathrm{x})=0,669\end{array}$ & $\begin{array}{l}\mathrm{k}_{\mathrm{obc}}=0,544 \\
\mathrm{u}_{5}(\mathrm{x})=0,955\end{array}$ \\
\hline
\end{tabular}

In cooperation of the co-evolution algorithm with the classifying system after performance of the calculation process the best solutions obtained from particular subpopulations the solutions are written into the classifier collection. The choice of the final solution variant depends upon the decision maker decider, who in this instance may be the operator managing the operation of the electric power Medium Voltage distribution network. 


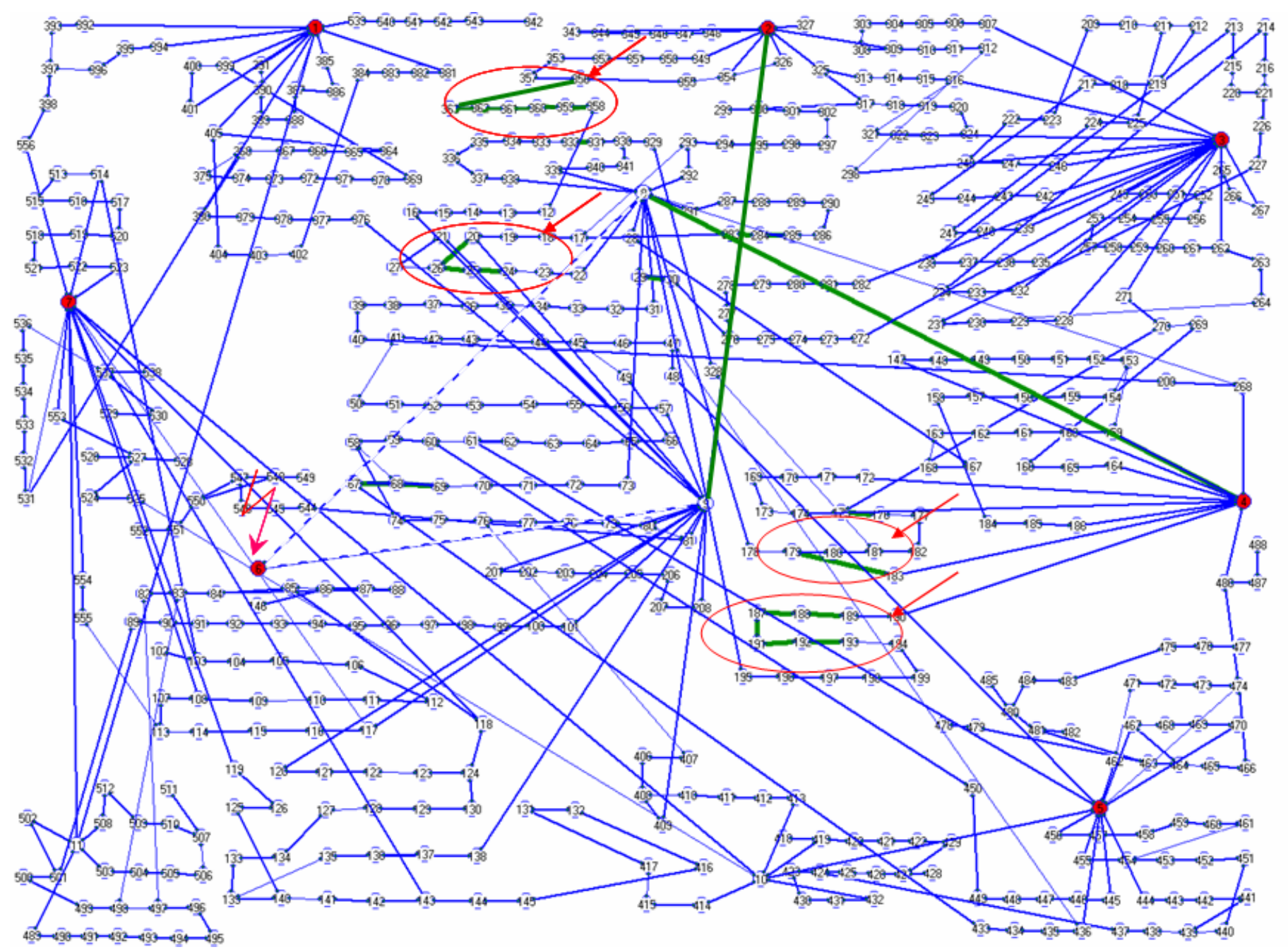

Fig. 6 Graph of the analysed distribution network with network configurations, being the best solution obtained in subpopulation 3

\section{CONCLUSION}

This article describes the development of this type of calculation methods, simultaneously containing their own innovative solution proposals concerning the application of a classification system working with the co-evolutionary algorithm. The calculations performed for the mapped real system of the medium voltage municipal distribution network of 556 nodes have given satisfactory results, confirming the adequate direction of the research. On the base of the results obtained so far the authors assume that the results can be further used in creation of decisive procedures for complex power electric systems management, taking the fault operation states into special consideration.

The method proposed by the author of the work is typified by the short time of designating the most rational post breakdown configurations in complex electric power Medium Voltage distribution network structures.

It is the use by the classifying system working with the coevolution algorithm that enables the effective creation of substitute scenarios for the Medium Voltage electric power distribution network. The method drawn up may be used in current systems managing the work of distribution networks to assist network operators in taking decisions concerning connection actions in supervised electric power systems.

\section{FUTURE SCOPE}

The proposed method fulfils the stated assumptions. It is typified by a short time of designation of substitute post breakdown configurations of configurations of complex medium voltage electric power distribution network structures. The drawn up method makes use of the classifying system cooperating with the co-evolutionary algorithm, which enables effective creation of substitute medium voltage distribution network configurations, for various network breakdown statuses.

It is thus a method based on systems that teach themselves. The method may be used in current systems and managing work on distribution networks to assist operators in making decisions concerning connection actions in supervised electric power systems.

The results obtained within the research performed, in the form of drawn up procedures to create the most effective configuration of the work of network appliances, may be used 
as elements of large very complex information systems used in intelligent electric power distribution networks. Application of integrated information systems assists distribution network management to optimise decision processes of system operator and in effect achieve the required level of services, by the minimisation of expenditure on network maintenance and appliances in a condition guaranteeing the supply of electric power of the required parameters.

\section{REFERENCES}

[1] C. C. Liu, S. J. Lee, S. S. Venkata, An expert system operational aid for restoration and loss reduction of distribution system. IEEE Trans. on Power Delivery, vol. 3, 1988, pp. 619-629.

[2] Y. Y. Hsu, M. Huang, Distribution system service restoration using a heuristic search approach. IEEE Trans. on Power Delivery, vol. 7, 1992, pp. 734-740.

[3] Y. Fukuyama, H. D. Chiang, Parallel genetic algorithm for service restoration in electric power distribution systems. Electric Power \& Energy Systems. vol. 18, no. 2, 1996, pp. 111-119.

[4] K. N. Miu, H. D. Chiang, B. Yuan, Fast service restoration for largescale distribution systems with priority customers and constraints. IEEE Trans. on Power Systems, vol. 13. no. 3, Aug. 1998, pp. 789-795.

[5] L. Morelato, A. J. Monticelli, Heuristic search approach to distribution system restoration. IEEE Trans. Power Delivery, vol. 4, Oct. 1989, pp. 2235-2241

[6] S. Wu. K. L. Tomsovic, C. S. Chen, A heuristic search approach to feeder switching operations for overload, faults, unbalanced flow and maintenance. IEEE Trans. Power Delivery. vol. 6. Oct. 1991, pp. 15791586.

[7] D. Shirmohammadi, Service restoration in distribution networks via network reconfiguration. IEEE Trans. Power Delivery, vol. 7, Apr. 1992, pp. 952-958.

[8] K. L. Butler, N. D. R. Sarma, R. Prasad, Network reconfiguration for service restoration in shipboard power distribution systems. IEEE Trans. Power Systems, vol. 16, Nov. 2001, pp. 653-661.
[9] Y. Hsiao, C. Chien, Enhancement of restoration service in distribution systems using a combination fuzzy-GA method. IEEE Trans. Power Systems, vol. 15, Nov. 2000, pp. 1394-1400.

[10] S. Toune, H. Fudo, T. Genji, Y. Fukuyama, Comparative study of modern heuristic algorithms to service restoration in distribution systems. IEEE Trans. Power Delivery, vol. 17, Jan. 2002, pp. 173-181.

[11] C. Chao-Shun, C-H. Lin, T. Hung-Ying, A rule-based expert system with colored petri net models for distribution system service restoration. IEEE Trans. Power Systems, vol. 17, Nov. 2002, pp. 1073-1080.

[12] S. Khushalani, J.M. Solanki, N.N. Schulz, Optimized Restoration of Unbalanced Distribution Systems. IEEE Transactions on Power Systems, no. 22, Issue 2. 2007, p. 624-630.

[13] Y. Kumar, B. Das, J. Sharma, Multiobjective, Multiconstraint Service Restoration of Electric Power Distribution System With Priority Customers. IEEE Transactions on Power Delivery, no. 23, Issue 1, 2008, p. 261-270.

[14] C. B. Delbem, A. C. P. L. F. Carvalho, N. G. Bretas, Main chain representation for evolutionary algorithms applied to distribution system reconfiguration. IEEE Trans. Power Systems., vol. 20, no. 1, Feb. 2005, pp. 425-436.

[15] K. Nara, A. Shiose, M. Kitagawa, T. Ishihara, Implementation of genetic algorithm for distribution systems loss minimum reconfiguration. IEEE Trans. Power Systems, vol. 7, no. 3, Aug. 1992, pp. 1044-1051.

[16] L. Augugliaro, F. R. Sanseverino, Multiobjective service restoration in distribution networks using an evolutionary approach and fuzzy sets. Elect. Power Energy Syst., vol.22, 2000, pp. 103-110.

[17] J. Stępień Z. Madej, Evaluation of structural redundancy efects in medium voltage cable networks. Rynek Energii No 4(83), 2009, pp. 5562

[18] J. Stępień: Changes in demand structure of energy carriers with the use of waste heat and renewable energy. Rynek Energii Issue: 5, OCT 2008, p. $58-62$

[19] J. Stępień, Evaluation of structural redundancy effects in medium voltage cable networks. Przegląd Elektrotechniczny Volume: 84 Issue: 4 2008, p. 128-131. 